\title{
Optical Illusions of Volume: Simulation- Based Form Finding and Fabrication
}

\author{
MARA MARCU, MING TANG, ADAM SCHUELER, HAN SHEN, \& MATTHEW BRUBAKER \\ University of Cincinnati
}

This paper presents alternatives to simulation driven processes which begin to exploit misbehaving behaviors in an ideal file to factory methodology. The work conducted together with students reconciles conflicting attitudes between traditional CAD/CAM strategies, interactive architecture, augmented reality and analog post-processing techniques. The projects mainly employ the use of a lightweight sheet material, parametric design, simulation, responsive architecture and investigate messier and more intuitive assembly strategies. Through this body of work we aim at developing techniques within the digital process that exploit a non-linear design and fabrication approach in which the hand and the machine become increasingly disrespectful of each other's artificially delineated boundary. By contaminating and continually disrupting each other, we believe that what are considered, by norm, flaws or faulty formations within the fabrication process can open up intriguing opportunities in architecture and design, otherwise missed.

\section{INTRODUCTION}

Simulation-based design has intrigued architects through a controlled process where prior empirical experience is augmented by the addition of data to drive design decisionmaking. This design process integrates interdisciplinary analysis and evaluative processes in an automated system that assists in designing better-performing buildings. In the practice of architecture, this process is based on various wellunderstood simulation methods such as modeling sunshade to respond to solar radiation data. Some of the emerging aspects of the architectural practice involve utilizing genetic algorithms in the design process, as well as performancedriven design to automatically generate an array of forms that respond to predefined rules. Through several courses taught at the University of Cincinnati, the authors explore how simulation-based computation is changing a static architectural form into an adaptive system that can respond to its performance. By not being limited by a strict linear workflow, where manually altering previous decisions is time-consuming and requires a regression of the design stage, architects are now able to establish generative workflows where multiple design aspects can be encoded as predefined rules.

\section{BACKGROUND}

Traditionally haute-couture designates high-end clothing specifically designed for a unique person's body and carefully hand-made with the utmost craft and dexterity. Contrary, prêt-à-porter collections are mass produced, machine-made and replicated to reach a wide pool of consumers. In the recent "Manus X Machina" exhibition at the Metropolitan Museum of Art, New York, the hand and the machine reconcile conflicting attitudes towards technology. Instead of exploiting their antagonism, the exhibition "suggests a spectrum of practice, whereby the hand and the machine are equal and mutual protagonists in solving design problems, enhancing design practices, and, ultimately, advancing the future of fashion" [1]. Reconciling the custom-made with the ready-made, a 2014 haute-couture wedding dress by Karl Lagerfeld for Chanel serves as a superlative example of the confluence between the two-"the pattern on the train was hand-painted with gold metallic pigment, machine-printed with rhinestones, and hand-embroidered with pearls and gemstones" [2].

Polarizing behaviors are also seen in architecture. In a recent lecture, Elizabeth Diller states "the speed of obsolescence makes technology a liability. Dumber is better than smarter, and the best thing to do for culture in the future is to secure real estate. It is as basic as that" [3]. Interdisciplinary in nature, this work distills these attitudes by asking the following questions. What is structure? How can skin and frame become a fluid continuum? What are the techniques that can describe the metamorphosis of two-dimensional, flat, sheet material to three-dimensional form? Can we develop a taxonomy of folds, bends, and joints? Can we bypass any in the process of assembly? Can the analog post digital processes be reinvigorated, and exploited as latent design opportunities in the digital era? What are the primitives of digital design? How do emergent technologies, on their immediate downfall to obsolesce, can mitigate the stringent need for securing real estate with contemporary aspirations?

\section{METHODS}

The focus of the methodology is placed on developing fullscale prototypes devoid of programmatic constraints, aimed at studying behaviors and tendencies while defining traits and formulating characters. Similarly, we obsessed over the dichotomy between the hand and the machine, while seeking breeding opportunities where each discipline can embrace practices of the other. To emphasize, analog interventions here were not seen as faulty digressions. By relaxing these otherwise necessary demands, we aimed at fostering a ludic and opportunistic environment to obsess over the questions mentioned above. Automated operations typical of CAD/ CAM strategies were employed. The workflow was 


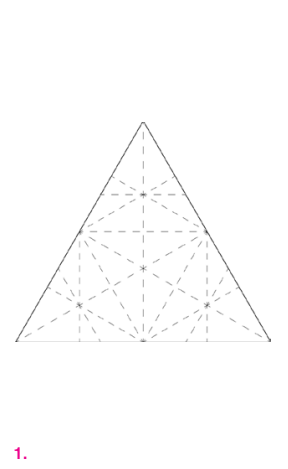

Figure 1: Base Unit Folding and Aggregation.

composed of fluidly navigating a process that encompasses working parametrically (in Digital Project/ CATIA), scripting, kinetics, virtual reality, and understanding constraint-driven design typical to sheet material fabrication (folding, bending and curving, material tolerances) and CNC outputs.

\section{PARAMETRIC SIMULATION}

"Bubbles" investigates the structural dichotomy between skin and frame through the study of a monocoque aggregation that allows the two to act as a fluid continuum. The project began as an exploration of patterns and inherent structures in conjunction with the tendencies and behaviors of material, in this case, flat sheets of matte polypropylene. Inspiration was drawn from two seemingly different yet intertwined sources: Islamic geometries and soap bubbles. The basis of any polygonal geometric pattern, no matter the complexity, can always be broken down into a series of triangles. Within these triangles lies an inherent logic by which they are organized and give rigidity to the assembly (Figure 1). Naturally, the triangles align themselves in a fashion where they can remain in a state of structural integrity. This same concept is explained at length in Self-made Tapestry: Pattern Formation in Nature by Philip Ball [4]. Ball uses the underlying structure of bubbles and honeycombs to describe how geometries found in nature arise from an innate desire for equilibrium. Specifically looking at these hexagonal forms, a similar structure is derived by taking a cue from Islamic ornaments.

Three site dependent armatures define the aggregation and modulation of the base unit in CATIA's Sheetmetal Workbench. This platform allowed us to simulate the folding process of flat sheet materials. Material constraints arose throughout the process relative to weight, strength, and translucency. One challenge in the process was the irregular nature of each triangle after being modulated. In its undisturbed state, the base unit is composed of equilateral triangles, which - when folded - allow for overlaps along a symmetric axis. However, after each unit has been customized, the asymmetry no longer facilitates the unrolling into a regular master triangle, which often had to be split into two separate pieces. Then, the location of connection holes was considered. Nylon
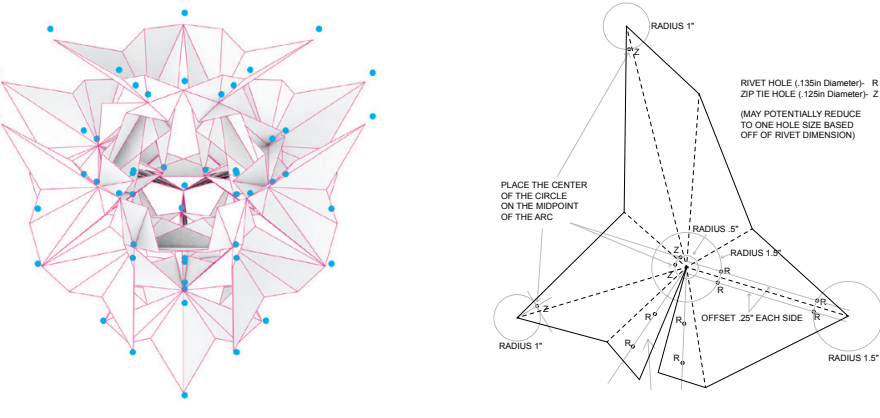

arrowhead rivets were used wherever faces overlapped, and zip-ties were employed wherever a hinge joint occurred. To reduce waste, all excess material implicit to the folding process - located at the center of each triangle unit - had to be removed. The installation was suspended in a sunken concrete courtyard which receives a varying degree of light throughout the day. Extrinsic (site related) parameters were considered to create an ephemeral experience that changed over the course of the day, encouraging onlookers to explore this interaction of light and form (Figure 4).

\section{INTERACTIVE SIMULATION}

Moving Monocoque began as an exploration into the possibility of an architecture that directly and autonomously responds to human needs and interactions. Central to the process was the question: can spaces become autonomously adaptive to address situational needs? The problem with most existing "multi-function" architecture is its lack of ability to respond to internal environmental needs. Externally architecture has begun to respond to climatic factors such as solar movement and heat gain throughout the day. Internally, however, most buildings remain static and ignorant of intrinsic (program and user related) transforming conditions.

A spatial canopy was constructed which responds to multidimensional user interaction and provides situational environments by utilizing sound sampling technology to drive a kinetic sequence, which in turn transforms the space in reaction to the amount of activity occurring underneath (Figure 2). The design process began with various site-specific modulation studies in the design development environment CATIA. It was determined, however, that for the specific benefit of smoothly functioning kinetics, as well as for a more structurally sound and spatial installation a single resilient base unit was superior. Grasshopper was used in unison with Kangaroo to simulate aggregation patterns and perform advanced physics testing to determine ideal layouts and kinetic possibilities.

The aggregation of the modular base unit is determined by applying the Catmull-Clark subdivision algorithm to the mesh surface of a polygon. This methodology enables the creation of smooth surfaces by dividing a given input surface 


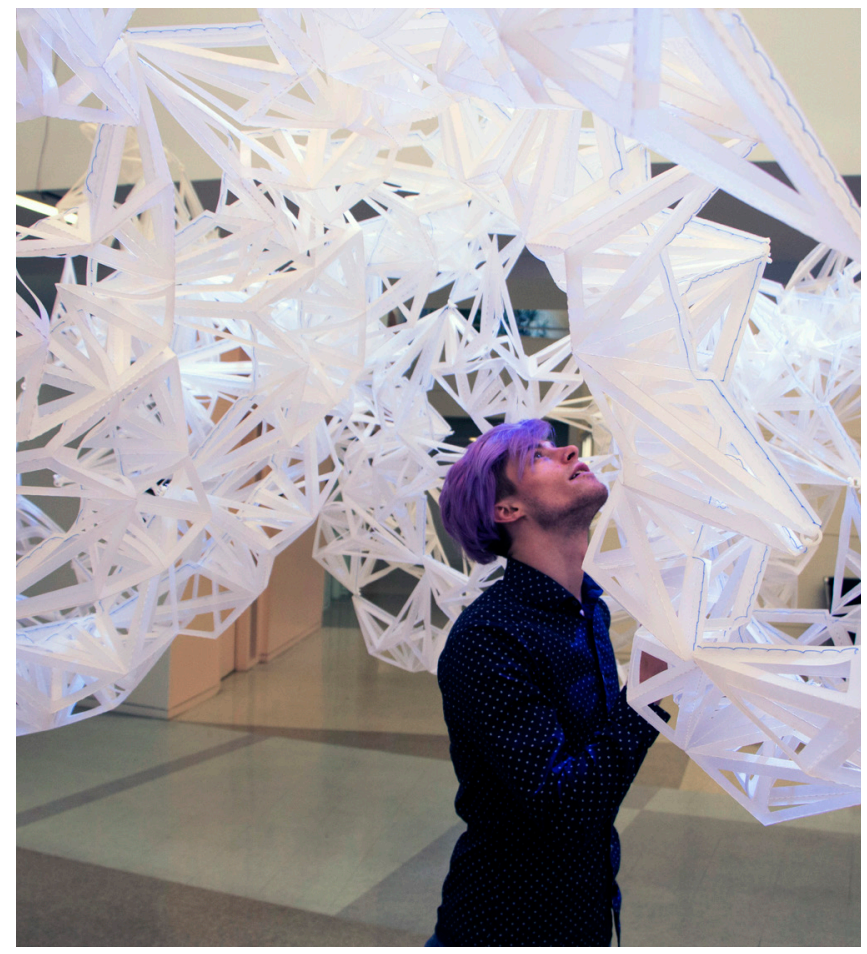

Figure 2: MO.MO., Detail.

recursively. One defining characteristic is that the resulting mesh surface consists of only quadrilaterals. [5] Because each of our cells has four arms that connect to identical arms of adjacent cells, a mesh surface that consists of quadrilaterals represents the ideal aggregation pattern, as each vertex on the surface can accurately represent a connection point between cells [6].

To simulate the outcome of each aggregation configuration when suspended and affected by step motors, Kangaroo was employed. While Kangaroo is capable of simulating the folding motion of each cell, the simulation of 96 fully modeled aggregated cells together proved to be too computation heavy to achieve timely and accurate results. Thus we utilized a simplified wireframe model by defining a series of computational "springs" on the input mesh in Kangaroo. These springs represent the pulling and pushing forces of each cell. These cells are attached to the wireframe in a state of equilibrium and are allowed to be affected by the simulated spring motion to assist in determining the mutation of each cell and its associated resiliency (Figure 3).

The Kangaroo simulation allowed us to test the outcome of each aggregation configuration quickly. A hexagonal mesh was chosen as the final form for its visual quality and topological complexity. Through the Kangaroo simulation, we determined that the topology of Catmull-Clark subdivided hexagon mesh has six ridges that extend from the center of the hexagon to each of the six corners. These ridges carry the weight of the adjacent valley to the support. By using the inherent physics of these ridges and valley, we can effectively change the shape of the entire canopy by applying an upward force to the ridges which in turn transfer the force down to the valley spines.

Arduino, a single-board microcontroller for building devices that interacts with the physical world, was used to control the motion of the canopy and the LED lights [7]. We opted to use NEMA17 stepper motor for motion and DMX enabled LED stage lights for lighting. To inform the Arduino board and activate the motor and lights, three condenser microphones are used to measure the ambient sound in the space. The three stepper motors are driven by three A3967 micro stepping drivers which through a tensile connection to the cells can move the installation in response to auditory input. Both the stepper motor driver and DMX LED lights require an un-interrupted signal stream to work. As Arduino boards run on a simple loop in which commands are executed in sequence, a second Arduino board is added to the system to send out two signal streams simultaneously. Once powered, the main board measures the sound level every millisecond for 500 milliseconds (onehalf second). After registering this sample the median value of each microphone input is then averaged with the adjacent microphones. By averaging the input values, the program can be closely controlled and prevented from being influenced by short bursts of loud sound. The result of this process is a more accurate ambient sound sample. The ambient sound values are then mapped to a three-dimensional Cartesian coordinate system, which determines the position of the stepper motors, thus influencing the height of each corner of the canopy along with the color of the LED lights.

To construct the units for the installation first the pattern is laser cut into a polypropylene sheet, and all fold lines are dashed in to allow for multi-directional bending. After cutting is complete, the two-dimensional patterns are then hand folded along the dashed cuts to create the three-dimensional unit. This unit is then sewn along the seams with 50-pound test monofilament line to cement the final three-dimensionality of the unit and to provide rigidity and resilience for each cell. These completed units were then connected to each other at each of the four tips in a radial pattern, each concentric ring containing more units than the previous until 96 units were conjoined to form the installation equilibrium shape determined by the previous simulations. Above where the installation hangs, a triangular wire structure was constructed out of the monofilament line to hold the motors and the form at the desired height. Once the wire structure was in place tensile members also constructed of monofilament line were employed to hoist and secure the geometry in place. Three of these tensile members are static and support the anchored corners of the installation. Three other tensile members run between the step motor pulley and the remaining three anchor points on the geometry enabling motion. 


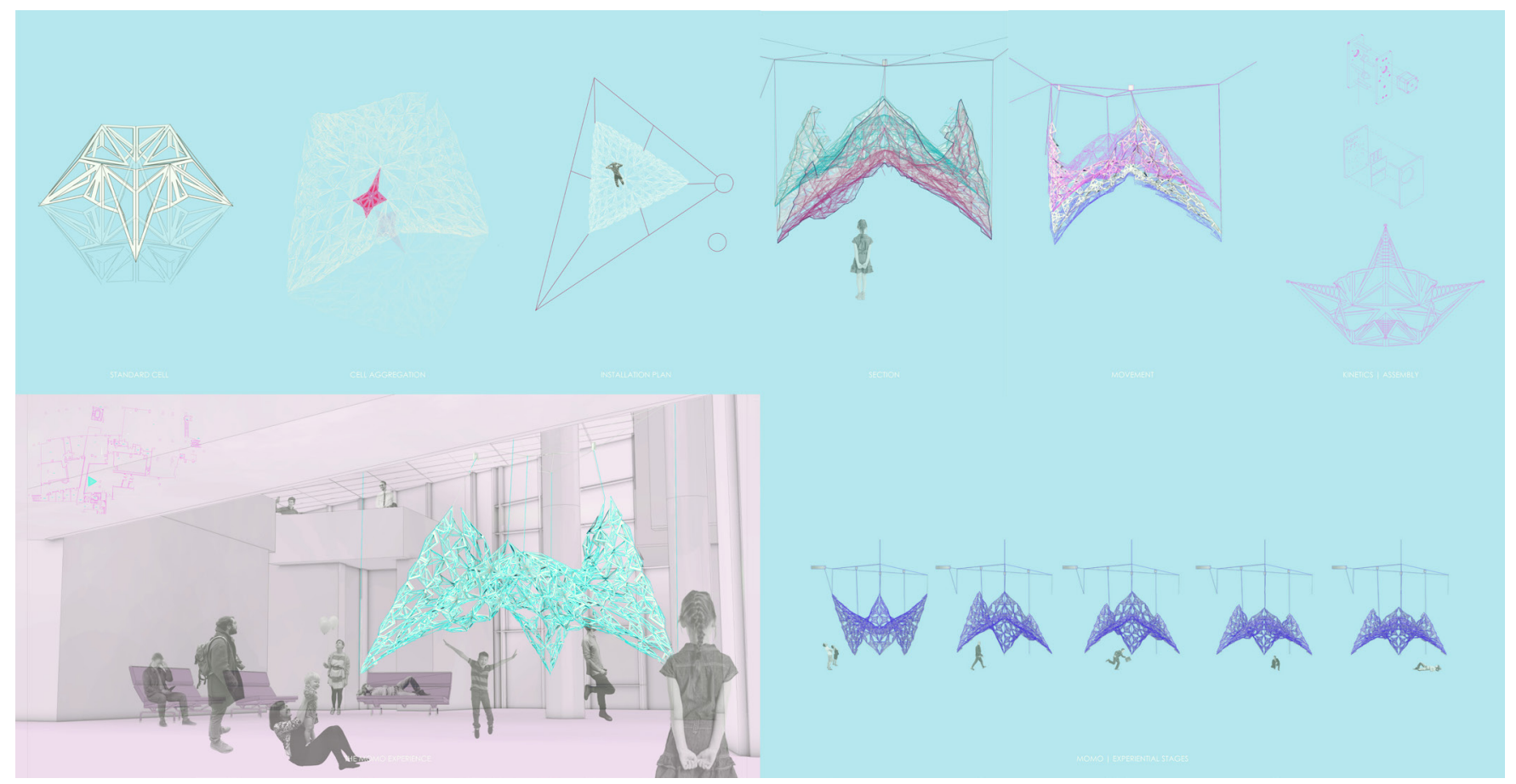

Figure 3: MO.MO.: Experiential Stages, Assembly, and Kinetics.

\section{AUGMENTED SIMULATION}

The Coral project is an installation that curates and expands on digital fabrication and mixed media work produced by the School of Architecture and Interior Design at the University of Cincinnati. The project is exhibited at the 2017 Sculpture Objects functional art and design (SOFA) expo in Chicago. The installation has two components. One constitutes several polyurethane surfaces using digital modeling, laser cutting techniques and rivet connections. These artifacts expand on the notion of skin and structure in architecture and collectively create a catalog of assembling possibilities.

The second part of the installation consists of several animated digital forms projected through Microsoft HoloLens augmented reality head-mounted display (HMD). The digital coral is reconstructed using marching cube algorithm to simulate the skeletal structure of a coral. It is animated using Autodesk Maya bone system and programmed to control by the user's gesture and voice through C\# scripts in the Unity game engine. The mixed reality coral installation act as a backdrop for the indexing of other sculptures during SOFA exhibition. The holographic animated coral and other visual effects are superimposed on the physical coral and to create an immersive undersea environment (Figure 5).

\section{CONCLUSION}

The projects speculate on the potential of future modes of structuring and inhabiting our world while examining approaches to digital and empirical simulation methodologies (specifically, spatial and structural).
Given the flexibility of polypropylene and the unpredictable structural qualities of variable connections, the fabrication at times relied heavily on empirical simulations. In certain cases, the dashed slits added adaptability and friction, while the method of 2D cut-sheet manipulation could yield far more complex degrees of modulation.

In other instances, the result of these decisions and the result of specific pedagogical adherence accompanied with intentional ignorance of carefully placed design principles allowed for an outcome which begins the conversation of how kinetic design can be better used to create adaptive spaces which respond directly and autonomously to the spatial needs of human interaction. If the work of Studio Roosegaarde on the Lotus 7 is examined, it is clear that the installation achieves automated kinetic movement. Equally as evident is the beauty and utility of the project and its impressive nature. The same can be said for "The Octopus Pavilion." Both installations utilize computational design to respond to human interaction, but both fail to create any type of experiential spatiality. The resulting product of a non-spatial but kinetic architecture project therefore usually inherits the form of a canopy, or of an object. These vernaculars function within themselves in response to outside factors but do not significantly alter the character or function of a contained usable space. In a way, these installations function similar to the kinetic façade systems being employed in contemporary architecture today. The lotus can be seen as an artistic translation of a system similar to that used in the design of the Al Bahar towers by Aedus and Arup. By applying the computational design ideas employed in the precedent projects to a monocoque structure the installation was better able to become a tool for space making and respond to environmental factors in a physical sense allowing for direct spatial influence on the user group. 

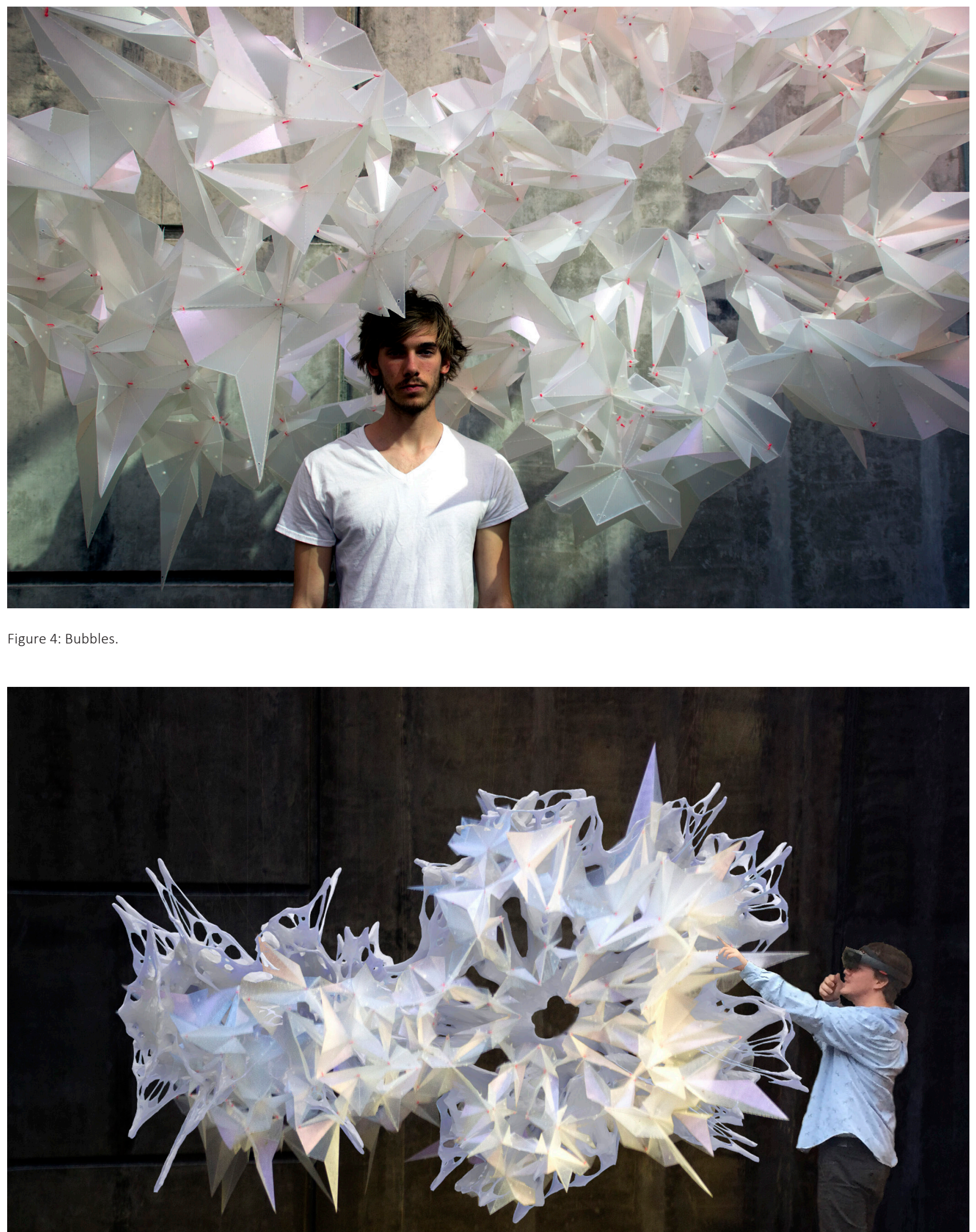
The scale of the space created in each motion state combined with the transient qualities during the transformation phase allowed for a space that was scaled perfectly for our ideal clients, children. The movement and use of lights also created playful and relatable situations, which intrigued and encouraged pausing and interaction. The installation was frequented by children of all ages who were intrigued by the pulsating movement, their ability to cause the space to react and the unique aesthetic quality of the installation. The main failure of the installation is its inability to form two separate conditions or spaces. The intent of creating an autonomously shifting architecture was not achieved in full. Due to restrictions in the programming and the materiality of the work instead of achieving two separate and unique spatial states, MO.MO. took on the characteristics of a constantly shifting space which by never fully forming a resting state generated infinite proxies of itself through its constant varying movement in response to the conditions beneath. This type of constant flux behavior resulted in a spatial reaction, which was not as true to creating space for activity, but is more of a continuous response to the situational activity. This result can be considered a success in the sense of autonomously responsive architecture. These results, however, are not yet succeeded in proving that computation design and kinetic architecture can provide a responsive and reactionary landscape to host different types of interaction.

These projects examined approaches where simulation methodologies were set and integrated into the fabrication pipeline to explore the potential optimization of various design-build solutions. The method extended to the spatial interaction of the environmental, or structural simulation and their controlled variables. Design optimization was accomplished through feeding design variables into a simulation, comparing results, and adjusting the output accordingly. We can conclude that the simulation-based design process has created an instability and de-centralization from a static form. Beginning with the analog form seeking methods by Gaudi and Otto, we can see a much more sophisticated process. The authors believe that the results expanded the boundary of conventional design through simulation and digital fabrication. These projects influenced students to think of form as a dynamic system where the identity and position of each element is a multiplied across a field of constraints. Here, the formal order of components is decentralized from the predetermined form. The form evolved in its relation to all other environmental, and structural elements of the system. Consequently, instead of thinking of form as the center, a simulation-based design has taught students to specify the process of evaluating before defining the formal elements' topology. Within simulation-based design, a form is now understood as a generative process of modulation that behaves singularly to the specific rule it has to adapt to.

\section{ENDNOTES}

1 Metropolitan Museum of Art, New York. (2016). "Manux x Machina: Fashion in the Age of Technology". Accessed Dec. 7, 2017. https://www.metmuseum.org/ exhibitions/listings/2016/manus-x-machina/exhibition-galleries

2 Metropolitan Museum of Art, New York. (2016). "Manux x Machina: Fashion in the Age of Technology". Accessed Dec. 7, 2017. https://www.metmuseum.org/ exhibitions/listings/2016/manus-x-machina

3 The Architect's Newspaper. (2017). "ACADIA 2016 showcased the diversity of cutting-edge computational design". Accessed Jan. 10, 2018. https://archpaper. com/2017/01/acadia-2016-recap/

4 Phillip Ball. "Bubbles". The Self-Made Tapestry: Pattern Formation in Nature. (New York: Oxford University Press Inc., 1999). 16-49.

5 Stam, J. (1998). "Exact evaluation of Catmull-Clark subdivision surfaces at arbitrary parameter values". Proceedings of the 25th annual conference on Computer graphics and interactive techniques - SIGGRAPH '98 (PDF). pp. 395-404. http://doi:10.1145/280814.280945. ISBN 0-89791-999-8.

6 Loop, C. and Schaefer, S. 2008. Approximating Catmull-Clark subdivision surfaces with bicubic patches. ACM Trans. Graph. 27, 1, Article 8 (March 2008), 11 pages. DOI = 10.1145/1330511.1330519. http://doi.acm. org/10.1145/1330511.1330519 6. Stam, J. (1998). "Exact evaluation of CatmullClark subdivision surfaces at arbitrary parameter values". Proceedings of the 25th annual conference on Computer graphics and interactive techniques -SIGGRAPH ‘98 (PDF). pp. 395-404. http://doi:10.1145/280814.280945. ISBN 0-89791-999-8.

7 Wikipedia. (n.d.). "Arduino". Accessed Nov. 28 2017. https://en.wikipedia.org/ wiki/Arduino. Arduino is an open source computer hardware and software company, project, and user community that designs and manufactures singleboard microcontrollers and microcontroller kits for building digital devices and interactive objects that can sense and control objects in the physical world.

\section{INSTALLATION CREDITS}

1. Bubbles: Adam Schueler, Peter Foster, Anousha Alamgir, Connor Tuthill. (University of Cincinnati students).

2. MO.MO.: Han Shen, Matthew Brubaker, Kiana Memarandadgar, Yasamin Enshaeian. (University of Cincinnati students). 